\title{
GLOBAL HOLOMORPHIC EXTENSION OF A LOCAL MAP AND A RIEMANN MAPPING THEOREM FOR ALGEBRAIC DOMAINS
}

\author{
Xiaojun Huang and Shanyu Ji
}

\section{Introduction}

In his paper of 1907 [Po07], Poincaré showed, among other things, that any local non-constant holomorphic map $f$ from an open piece of the sphere $\partial \mathbb{B}^{2}$ into $\partial \mathbb{B}^{2}$ extends to a global biholomorphic map between the ball $\mathbb{B}^{2}$. This result was generalized to $\mathbb{B}^{n+1}$ by Tanaka [Ta62] and was further established in a much more general setting by Alexander [A74]. Since then, the mixed equivalence problem was naturally formulated (see for instance [Wel82], [Be90]): How far can a local equivalence map between the boundaries of two nice domains be biholomorphically extended? A significant contribution along the lines of this direction was made by Webster: In [We77], he not only extended Poincaré's theorem to real ellipsoids, but also did a pioneer work by showing a type of Chow's theorem for local maps between algebraic domains. In particular, he showed that any local equivalence map between two smooth algebraic domains extends as a branched algebraic map. Webster's algebraicity theorem was established in more general situations in the recent deep work of Baouendi, Ebenfelt and Rothschild (see [BR95], [BER96]), and in the first author's work ([Hu94], [Hu94T]). In [CJ96], Chern and the second author showed a related result: Any local map $f$, which maps a piece of $\partial D$ into $\partial \mathbb{B}^{n+1}$, extends along any path $\gamma \subset \bar{D}$ as a locally bimeromorphic map, where $D$ is a bounded domain with real analytic spherical boundary.

The purpose of this paper is to study the global holomorphic extension of a local map between algebraic domains, i.e, domains whose boundaries are locally defined by real polynomials. Our first result is the following theorem:

Theorem 1.1. Let $D, D^{\prime} \subset \mathbb{C}^{n+1}$ be bounded domains with algebraic smooth boundaries $\partial D$ and $\partial D^{\prime}$. Let $a \in \partial D$, and $U_{a}$ a neighborhood of a in $\mathbb{C}^{n+1}$. Let $f: U_{a} \rightarrow f\left(U_{a}\right) \subset \mathbb{C}^{n+1}$ be a biholomorphic map such that $f\left(U_{a} \cap \partial D\right) \subset \partial D^{\prime}$.

Received January 29, 1998.

The first author acknowledges the support from NSF DMS-9500881 and an NSF postdoctoral fellowship; while the second author acknowledges the support of NSF DMS-943761 
Suppose that $D^{\prime}$ is strongly pseudoconvex and $\partial D$ is connected. Then, for any given path $\gamma(t) \in \bar{D}, 0 \leq t \leq 1$, with $\gamma(0)=a$, the local map

$$
f \text { extends holomorphically along } \gamma \text {. }
$$

One of the main points of Theorem 1.1 is that the strong pseudoconvexity on $\partial D$ is not required. Nevertheless, the condition of strong pseudoconvexity on the target space $D^{\prime}$ is important. (However, see Remark 6.1 (b)). In fact, the local map, $(z, w) \mapsto(z, \sqrt{w})$, from a small piece of $\partial \mathbb{B}^{2}$ near $(0,1)$ into the boundary of the egg domain $\left\{|z|^{2}+|w|^{4}-1=0\right\}$, cannot be extended along some paths in $\partial \mathbb{B}^{2}$. Also, it should be mentioned that the algebraicity assumption in Theorem 1.1 is crucial. In fact, Burns and Shnider [BS76] had examples showing that there are many local biholomorphic maps between the ball and a certain real analytic (strongly pseudoconvex) spherical domain $D$ which cannot be holomorphically extended along some path $\gamma$ inside $\partial \mathbb{B}^{n+1}$.

If the path $\gamma \subset \partial D$ and if one puts the strong pseudoconvexity on $\partial D,(1.1)$ was already proved (without the algebraic condition) in the following special cases by Pinchuk and Vitushkin-Ezhov-Kruzhilin: (i) $\partial D^{\prime}=\partial \mathbb{B}^{n+1}$ and $\partial D$ is spherical real analytic [Pi78]; and (ii) both $\partial D$ and $\partial D^{\prime}$ are non-spherical strongly pseudoconvex real analytic [Pi78], [VEK86]. By the way, the proofs of those two results were completely separated, depending on the non-spherical and spherical cases. However, our method, different from theirs, deals with both cases simultaneously.

As an application of Theorem 1.1, we obtain immediately the following Riemann Mapping Theorem in the algebraic category. Recall that a real analytic domain $D$ is called a spherical domain if for any point $p \in \partial D$, there is an open neighborhood $U$ of $p$ in $\mathbb{C}^{n+1}$ and a biholomorphic map $\Phi$ defined over $U$ such that $\Phi(U \cap \partial D) \subset \partial \mathbb{B}^{n+1}$ and $\Phi(U \cap D) \subset \mathbb{B}^{n+1}$.

Theorem 1.2. Any bounded algebraic spherical domain $D \subset \mathbb{C}^{n+1}$ must be biholomorphic to the unit ball $\mathbb{B}^{n+1}$.

The surprising feature of Theorem 1.2 is that there is no need to impose any topological restriction on $D$. (See also Theorem $1.2^{\prime}$ stated at the end of the paper). Meanwhile, there are many bounded real analytic spherical domains in $\mathbb{C}^{n+1}$, which are not biholomorphic to the ball $\mathbb{B}^{n+1}$, by the famous examples of Burns and Shnider [BS76]. Apparently, the theorem fails in the setting of one complex variable case. To our knowledge, we do not know any other versions of the Riemann mapping theorem which hold for algebraic domains but not for real analytic domains ([Wo77], [Ro80], [CJ96]). (The reader may like to compare this theorem with certain other work done in [BHR96], [E96], where the algebraicity also plays an essential role.)

As some other applications of Theorem 1.1, we give here the following two more corollaries: 
Corollary 1.3. Let $D, D^{\prime}, U_{a}, f, \gamma$ be as in Theorem 1.1. If, in addition, $\partial D$ is also strongly pseudoconvex, then the extension $f$ along $\gamma$ is locally biholomorphic. Moreover, if $\gamma(t) \in D$ for $0<t<1$, then

$$
f(\gamma(t)) \in D^{\prime} \quad \text { for } 0<t<1 \text {. }
$$

Corollary 1.4. Let $D, D^{\prime}, U_{a}, f$ be as in Corollary 1.3. (i) If, in addition, $D$ is simply connected, the local map $f$ then extends to a covering map $f: D \rightarrow D^{\prime}$. (ii) If, in addition, $D$ and $D^{\prime}$ are both simply connected, then the local map $f$ extends to a biholomorphic map $f: D \rightarrow D^{\prime}$.

Our proof of Theorem 1.1 is based on the technique of Segre family theory (cf. [We77], [We78], [DFY94], [Hu94], [BER96], [Hu96]). First, by a celebrated theorem of Webster [We77], the map under study is algebraic. However, apriori, the branch locus $\mathcal{E}$ of $f$ may cut $\bar{D}$. Consider $\gamma \subset \partial D$ and suppose that $f$ extends along $\gamma(t)$ for $0 \leq t<c \leq 1$. Suppose that $p=\gamma(c) \in \partial D \cap \mathcal{E}$. There are two different cases that we have to study separately. The first case is when $\mathcal{E}$ cuts only the boundary $\partial D$ at $p$ but not inside $D$. Then, $f$ extends inside $D$ near $p$ and we can extend the graph of $f$ in terms of Segre varieties over the space outside of $D$ near $p$. Using the strong pseudoconvexity of $\partial D^{\prime}$, we then make the desired single-valued extension. The second case is when $\mathcal{E}$ cuts $\partial D$ in both sides at $p$. By passing to a nearby point, we can then assume that $p$ is a smooth point of $\mathcal{E}$ and $\mathcal{E}$ is transversal to $\partial D$ at $p$, too. Therefore, the fundamental group of $U-\mathcal{E}$ is isomorphic to $\mathbb{Z}$ and is thus generated by a simple loop around $\mathcal{E}$. Using the Segre variety $Q_{z}$, we can connect any two branches of $f$ so that they are forced to be the same by the strong pseudoconvexity on $\partial D^{\prime}$.

In this paper, we denote by $\bar{z}$ the complex conjugate of a complex number z. For a set $A \subset \mathbb{C}^{n+1}$, we denote by $\bar{A}$ its topological closure. For a point $p \in \mathbb{C}^{n+1}$, we denote by $U_{p}$ and $\mathcal{P}_{p}$ small neighborhoods of $p$ in $\mathbb{C}^{n+1}$ with $\mathcal{P}_{p} \subset \subset U_{p}$. Let $\gamma$ be a path in $\mathbb{C}^{n+1}$ and $h$ a holomorphic map defined near $\gamma(0)$. In all that follows, we say that $h$ extends holomorphically along $\gamma$ if there are finitely many pairs $\left\{U_{i}, h_{i}\right\}_{i=0}^{k-1}$ such that (a) each $U_{i}$ is geometrically convex, $h_{i}$ is holomorphic over $U_{i}$ and $h_{0}=h$ near a small neighborhood of $\gamma([0,1 / k])$; (b) for $0 \leq i \leq k-1, \gamma([i / k,(i+1) / k]) \subset \subset U_{i}$; and (c) $h_{i}=h_{i+1}$ over $U_{i} \cap U_{i+1}$. For simplicity, after defining some notations on the source space, we add 'primes' for the corresponding notations on the target space, unless stated explicitly.

\section{More notation, definitions and preliminaries}

Let $D$ be as in Theorem 1.1. For any point $p \in \partial D$, we can choose a realvalued real polynomial $r$ and a small open ball $U_{p}$ centered at $p$ such that $\partial D \cap U_{p}=\left\{z \in U_{p}: r(z, \bar{z})=0\right\}$ and $\left.d r\right|_{\partial D \cap U_{p}} \neq 0$. For any $z \in U_{p}$, the subvariety in $U_{p}$

$$
Q_{z}:=\left\{w \in U_{p} \mid r(w, \bar{z})=0\right\}
$$


is called the Segre variety of $\partial D$ associated to $z$. Shrinking $U_{p}$ and choosing another sufficiently small ball $\mathcal{P}_{p}$ centered at $p$, we may assume that for each $z \in \mathcal{P}_{p}, Q_{z}$ is a connected complex submanifold and $U_{p}-\partial D$ has two simply connected components.

For any $z \in \mathcal{P}_{p}$, we define a subvariety $A_{z}:=\left\{w \in \mathcal{P}_{p}: Q_{w}=Q_{z}\right\}$. Since $\partial D$ is compact and real analytic, it is known [DFY94] that when $\mathcal{P}_{p}$ is sufficiently small,

$$
\# A_{z}<\infty, \quad \forall z \in \mathcal{P}_{p}
$$

Shrinking $\mathcal{P}_{p}$ again, we can find a smooth conjugating operator (cf. [DFY94], [Hu96]) $\mathcal{R}: \mathcal{P}_{p} \rightarrow U_{p}$, such that $z^{*}:=\mathcal{R}(z) \in Q_{z}, \mathcal{R}^{2}(z)=z, \mathcal{R}\left(\mathcal{P}_{p}\right) \subset \subset U_{p}$, $\mathcal{R}$ reverses the sides of $\partial D$, and $\left.\mathcal{R}\right|_{\partial D}=i d$. In what follows, we always arrange the pair $\left\{U_{p}, \mathcal{P}_{p}\right\}$ such that the above mentioned properties hold.

For any $z \in \mathcal{P}_{p} \backslash \bar{D}$, we denote by $Q_{z}^{c}$ the irreducible component of $Q_{z} \cap D$ which contains the point $z^{*}$.

Consider the biholomorphic map $f: U_{a} \rightarrow f\left(U_{a}\right)$ with $f\left(U_{a} \cap \partial D\right) \subseteq \partial D^{\prime}$ as in Theorem 1.1. Since $\partial D$ and $\partial D^{\prime}$ are algebraic, by a result of Webster [We77], $f$ is an algebraic map. Hence its graph $\Gamma_{f}:=\left\{(z, f(z)) \mid z \in U_{a}\right\}$ extends to an irreducible complex $(n+1)$-dimensional projective algebraic subvariety

$$
\mathcal{V} \subset \mathbb{P}^{n+1} \times \mathbb{P}^{n+1}
$$

Denote by $\pi$ and $\pi^{\prime}$ the restriction of the following natural projections to $\mathcal{V}$, respectively:

$$
\mathbb{P}^{n+1} \times \mathbb{P}^{n+1} \rightarrow \mathbb{P}^{n+1},(z, w) \mapsto z, \text { and } \mathbb{P}^{n+1} \times \mathbb{P}^{n+1} \rightarrow \mathbb{P}^{n+1},(z, w) \mapsto w .
$$

Notice that there are complex algebraic subvarieties $\mathcal{E}, \mathcal{S} \subset \mathbb{C}^{n+1}$ such that for any path $\gamma \subset \mathbb{C}^{n+1}$ with $\gamma(0)=a$ and $\gamma \cap \mathcal{E}=\emptyset, f$ extends holomorphically along $\gamma$, and $f$ extends biholomorphically along $\gamma$ when $\gamma \cap(\mathcal{E} \cup \mathcal{S})=\emptyset$. Since there is a certain subvariety $\mathcal{E}^{*}$ of $\mathcal{V}$ such that the restrictions of $\pi$ and $\pi^{\prime}$ to $\mathcal{V} \backslash \mathcal{E}^{*}$ are locally biholomorphic, it can be easily seen that for any irreducible subvariety $E$ in $\mathbb{P}^{n+1}$ with positive codimension, $\pi^{\prime}\left(\pi^{-1}(E)\right)$ is also a subvariety of $\mathbb{P}^{n+1}$ with positive codimension.

Let $\widetilde{p} \in \partial D$ such that $f: U_{\widetilde{p}} \rightarrow f\left(U_{\widetilde{p}}\right)$ is holomorphic. Write $z^{\prime}=f(z), \overline{z^{\prime}}=$ $\overline{f(z)}=\bar{f}(\bar{z})$. When $U_{\widetilde{p}}$ is sufficiently small, we have

$$
r^{\prime}(f(z), \bar{f}(\bar{w}))=u(z, \bar{w}) r(z, \bar{w}), \quad \forall z, w \in U_{\widetilde{p}},
$$

with $u$ holomorphic in $(z, \bar{w})$. Hence for any $w \in U_{\widetilde{p}}$, we see the following well-known invariant property [We77]

$$
f\left(Q_{w}\right) \subseteq Q_{f(w)}^{\prime}
$$


Next, let $p \in \partial D, \mathcal{R}, \mathcal{P}_{p}$ and $U_{p}$ be as introduced before. Suppose that $U \subset \subset \mathcal{P}_{p}$ is an open subset such that $U \cap \partial D \neq \emptyset$ and $f: U \rightarrow f(U) \subset \mathbb{C}^{n+1}$ is holomorphic. (Notice here that $f$ is not assumed apriori to be holomorphic over $\left.U_{p}\right)$. Let $\theta(t) \in \mathcal{P}_{p}, 0 \leq t \leq 1$, be a path with $\theta(0) \in U \cap \partial D$. Suppose that $f$ extends along both paths $\theta$ and $\theta^{*}:=\mathcal{R}(\theta)$ holomorphically. Denote by $f_{\theta(t)}$ and $f_{\theta^{*}(t)}$ for the germs of the holomorphic continuation of $f$ along $\theta$ and $\theta^{*}$ at the points $\theta(t)$ and $\theta^{*}(t)$, respectively. For simplicity, we also occasionally write $f_{\theta}:=f_{\theta(t)}$ and $f(\theta(t)):=f_{\theta(t)}(\theta(t))$, when there is no risk of causing confusion. For each point $z \in \mathbb{C}^{n+1}$, we write, in what follows, $O_{z}$ or $O(z)$ for a small ball centered at $z$, whose size may be different in different contexts.

Lemma 2.1. With the above notation and assumption, suppose further that $\theta(t)$ $(0 \leq t \leq 1)$ is a Jordan path. Then one has the following invariant property for the extension of $f$ :

$$
\begin{aligned}
f_{\theta^{*}(t)}\left(O_{\theta^{*}(t)}\right. & \left.\cap Q_{\theta(t)}\right) \subseteq \\
Q_{f(\theta(t))}^{\prime} & ; \text { and } f_{\theta(t)}\left(O_{\theta(t)} \cap Q_{\theta^{*}(t)}\right) \subseteq Q_{f\left(\theta^{*}(t)\right)}^{\prime}, \quad 0 \leq t \leq 1 .
\end{aligned}
$$

Proof of Lemma 2.1. As above, write $\rho$ and $\rho^{\prime}$ for the algebraic defining functions of $D$ and $D^{\prime}$ (near $p$ and $p^{\prime}$ ), respectively. Let $\Omega(\theta) \subset \mathcal{P}_{p}$ be a sufficiently small simply connected neighborhood of the open arc $\theta((0,1))$ such that $f_{\theta}$ extends holomorphically to $\Omega(\theta)$ and $f_{\theta^{*}}$ extends holomorphically to $\mathcal{R}(\Omega(\theta)$ ).

Consider the function: $\Sigma(z, \bar{w}):=\rho^{\prime}\left(f_{\theta}(z), \overline{f_{\theta^{*}}(w)}\right)$, where

$$
(z, \bar{w}) \in \mathcal{M}^{*}=(\Omega(\theta) \times \overline{\mathcal{R}(\Omega(\theta))}) \cap \mathcal{M} .
$$

Here $\mathcal{M}:=\{(z, \bar{w}): \rho(z, \bar{w})=0\}$ can be assumed to be a connected complex manifold with coordinates in $(z, \bar{w})$. Then $\Sigma$ is clearly a single-valued function holomorphic in $(z, \bar{w})$. (2.5) is now equivalent to saying that $\Sigma(z, \bar{w}) \equiv 0$, when $(z, w)\left(\in \mathcal{M}^{*}\right) \approx\left(\theta(t), \theta^{*}(t)\right)$ with $t<<1$. Hence, it follows that $\Sigma(z, \bar{w}):=$ $\rho^{\prime}\left(f_{\theta}(z), \overline{f_{\theta^{*}}(w)}\right) \equiv 0$ over the connected neighborhood of $\left\{\left(\theta(t), \theta^{*}(t)\right): t \in\right.$ $(0,1)\}$ in $\mathcal{M}^{*}$. This is equivalent to saying that $(2.6)$ holds for $0 \leq t<1$. Passing to the limit, we similarly prove that it also holds at $t=1$. The proof is complete.

Finally we state a quite standard result whose proof, for example, can be found in [We 79]. Notice that Lemma 2.2 is the only place we use the restriction of the strong pseudoconvexity (rather than the weak pseudoconvexity) for the proof of Theorem 1.1.

Lemma 2.2. Let $p^{\prime} \in \partial D^{\prime}$ be a strongly pseudoconvex point and $U_{p^{\prime}}^{\prime}$ a sufficiently small neighborhood of $p^{\prime}$ in $\mathbb{C}^{n+1}$ as introduced before. Then for any two points $w_{1}, w_{2} \in \mathcal{P}_{p^{\prime}}^{\prime}, Q_{w_{1}}^{\prime}=Q_{w_{2}}^{\prime}$ holds if and only if $w_{1}=w_{2}$. 


\section{Some local properties of the extension of $f$}

Lemma 3.1. Let $D, D^{\prime}, f, U_{a}$ be as in Theorem 1.1. Let $\gamma(t) \in \partial D, 0 \leq t \leq 1$, be a path with $\gamma(0)=a$. Suppose that $f$ extends along $\gamma(t) \in \partial D, 0 \leq t<c \leq 1$, holomorphically and that $f\left(\gamma\left(t_{j}\right)\right) \rightarrow p^{\prime} \in \partial D^{\prime}$ for some sequence $t_{j} \nearrow c$. Write $p:=\gamma(c)$. Then for any $\epsilon>0$, there is a certain small constant $\delta>0$ such that for any path $\sigma(t) \in B(p, \delta)-\mathcal{E} \cup \mathcal{S} \cup \mathcal{R}(\mathcal{E} \cup \mathcal{S}), 0<t \leq 1$, with $\sigma(0)=\gamma\left(c_{1}\right)$ for some $0<c_{1}<c$, the extended map $f$ along $\sigma$ (with the initial value $f_{\gamma\left(c_{1}\right)}$ ) takes value in $B^{\prime}\left(p^{\prime}, \epsilon\right)$.

Proof of Lemma 3.1. First, by the monodromy theorem and some simple topological arguments, it is clear that we can assume, without loss of generality, that the $\sigma$ in the lemma is a Jordan path.

Suppose that the above statement is not true. For any $\epsilon>0$ with $B^{\prime}\left(p^{\prime}, \epsilon\right) \subset \subset$ $\mathcal{P}_{p^{\prime}}^{\prime}$, then when $\delta>0$ is sufficiently small, one can find easily a Jordan path $\sigma_{\epsilon_{\delta}}(t) \in B(p, \delta)-\mathcal{E} \cup \mathcal{S} \cup \mathcal{R}(\mathcal{E} \cup \mathcal{S})(0<t \leq 1)$ such that (a) $f_{\sigma_{\epsilon_{\delta}}}\left(\sigma_{\epsilon_{\delta}}(t)\right) \in B^{\prime}\left(p^{\prime}, \epsilon\right)$ for $0 \leq t<1$, (b) $\sigma_{\epsilon_{\delta}}(0)=\gamma\left(t_{\epsilon_{\delta}}\right)$ for some $t_{\epsilon_{\delta}}$ with $0<t_{\epsilon_{\delta}}<c$ and $\gamma\left(t_{\epsilon_{\delta}}\right) \in \mathcal{P}_{p}$, and $(\mathrm{c})$

$$
f_{\sigma_{\epsilon_{\delta}}(1)}\left(\sigma_{\epsilon_{\delta}}(1)\right) \in \partial B^{\prime}\left(p^{\prime}, \epsilon\right),
$$

where $f_{\sigma_{\epsilon_{\delta}}}$ is the holomorphic extension of $f$ (with initial value $f_{\gamma\left(t_{\epsilon_{\delta}}\right)}$ ) along $\sigma_{\epsilon_{\delta}}$.

Since $\sigma_{\epsilon_{\delta}}((0,1]) \cap(\mathcal{E} \cup \mathcal{S} \cup \mathcal{R}(\mathcal{E} \cup \mathcal{S}))=\emptyset, \sigma_{\epsilon_{\delta}}^{*}((0,1]) \subset U_{p}-\mathcal{E} \cup \mathcal{S}$ and $f$ extends along both $\sigma_{\epsilon_{\delta}}$ and $\sigma_{\epsilon_{\delta}}^{*}$ biholomorphically. By (2.5), we conclude that

$$
f_{\sigma_{\epsilon_{\delta}}^{*}(1)}\left(Q_{\sigma_{\epsilon_{\delta}}(1)} \cap O_{\sigma_{\epsilon_{\delta}}^{*}(1)}\right) \subseteq Q_{f\left(\sigma_{\epsilon_{\delta}}(1)\right)}^{\prime}
$$

Hence, it yields that $\pi^{\prime}\left(\pi^{-1}\left(\hat{Q}_{\sigma_{\epsilon_{\delta}}(1)}\right)\right) \supset Q_{w_{\epsilon_{\delta}}}^{\prime}$ with $w_{\epsilon_{\delta}}=f\left(\sigma_{\epsilon_{\delta}}(1)\right) \in \partial B\left(p^{\prime}, \epsilon\right)$. Here, for $z \approx p$, we use $\hat{Q}_{z}$ to denote the following algebraic compactification of $Q_{z}$ in $\mathbb{P}^{n+1}$ : Write the defining function $r$ of $\partial D$ near $p$ as $r(\eta, \bar{z})=$ $\sum_{\alpha,|\alpha|=0}^{N} a_{\alpha}(\bar{z}) \eta^{\alpha}$ with $\eta=\left(\eta_{1}, \cdots, \eta_{n+1}\right)$. Then $\hat{Q}_{z}=\left\{\left[\eta_{1}, \cdots, \eta_{n+2}\right] \in \mathbb{P}^{n+1}\right.$ : $\left.\sum_{\alpha=\left(\alpha_{1}, \cdots, \alpha_{n+1}\right) ;\|\alpha\|=0}^{N} a_{\alpha}(\bar{z}) \eta_{1}^{\alpha_{1}} \cdots \eta_{n+1}^{\alpha_{n+1}} \eta_{n+2}^{N-\|\alpha\|}=0.\right\}$

Letting $\delta \rightarrow 0$ and letting $w_{\epsilon}$ be a limit point of $\left\{w_{\epsilon_{\delta}}\right\}$, it gives that $\pi^{\prime} \circ$ $\pi^{-1}\left(\hat{Q}_{p}\right)$ contains $Q_{w_{\epsilon}}^{\prime}$. Notice that $w_{\epsilon} \in \partial B^{\prime}\left(p^{\prime}, \epsilon\right)$ and $\pi^{\prime} \circ \pi^{-1}\left(\hat{Q}_{p}\right)$ can only have finitely many irreducible components (each of which has positive codimension, by the previous observation.) Varying $\epsilon$, we conclude that there are infinitely many $w_{\epsilon_{k}} \rightarrow p^{\prime}$, such that $w_{\epsilon_{k}} \in \partial B^{\prime}\left(p^{\prime}, \epsilon_{k}\right), \epsilon_{k} \searrow 0$, and that all $Q_{w_{\epsilon_{k}}}^{\prime}$ are the same near $p$. This contradicts (2.2).

In order to prove Theorem 1.1, we need Lemma 3.2 below.

Lemma 3.2. Let $p \in \partial D \cap E$, where $E$ is a smooth complex hypersurface in $U_{p}$ such that $E$ intersects both sides of $\partial D \cap U_{p}$. Write $G=\partial D \cap E \cap U_{p}$. Then $E$ intersects $\partial D$ transversally along an open dense subset of $E \cap \partial D$.

Proof of Lemma 3.2. We first notice that $\partial D$ is pseudoconvex. Indeed, let $\mathcal{E}$ and $\mathcal{S}$ be as defined before. Then, we know that $f$ extends locally biholomorphically 
along any path without cutting $\mathcal{S}^{*}:=\mathcal{E} \cup \mathcal{S}$. Clearly, $\mathcal{S}^{*}$ has no open piece inside $\partial D$, for $\partial D$ is a compact real algebraic hypersurface. Hence $\mathcal{S}^{*} \cap \partial D$ has real codimension at least 2 in $\partial D$. That is, $\partial D \backslash \mathcal{S}^{*}$ is connected. Hence, $\partial D$ must be strongly pseudoconvex over $\partial D \backslash \mathcal{S}^{*}$, for $\partial D \backslash \mathcal{S}^{*}$ is locally equivalent to its image in $\partial D^{\prime}$, which we assumed to be strongly pseudoconvex. Therefore, one can easily conclude that $D$ is strongly pseudoconvex at points away from $\partial D \cap \mathcal{S}^{*}$, and is pseudoconvex at points in $\partial D \cap \mathcal{S}^{*}$.

Next, notice that the hypothesis indicates that $E \cap \partial D$ is of real codimension 1 in $E$, for $\partial D$ separates $E$. (See [Ru80].) Since $E \cap \partial D$ is a real analytic set, we see that $E \cap \partial D$ is smooth with real codimension 1 in $E$ almost everywhere.

Let now $q$ be a smooth point of $\partial D \cap E \cap U_{p}$. Since $D$ is pseudoconvex, we can assume, by a result of Diederich-Fornaess [DF77], that $D$ is defined near $q$ by a smooth function $\rho^{*}$ such that $-\left(-\rho^{*}\right)^{2 / 3}$ is plurisubharmonic over $D \cap O_{q}$.

On the other hand, $E \cap \partial D$ has codimension 1 in $E$ near $q$. Restricting $-\left(-\rho^{*}\right)^{2 / 3}$ to $E \cap D$ near $q$ and applying the Hopf lemma, we conclude that the lower limit of the normal derivative, with respect to $\partial D \cap E$, of $-\left(-\left.\rho^{*}\right|_{E}\right)^{2 / 3}$ at $q$ is non zero. This then infers that $\left.\mathrm{d}\right|_{E} \rho^{*} \neq 0$ at $q$. Namely, $E$ cuts $\partial D$ transversally at $q$. The proof of lemma 3.2 is thus complete.

Remark 3.3. (a) For Lemma 3.1, the pseudoconvexity assumption of $D^{\prime}$ (and consequently, of $D$ ) is redundant. However, for Lemma 3.2, the pseudoconvexity of $D$ is crucial.

(b) When the $r(z, \bar{z})$ in the proof of Lemma 3.1 is just assumed to be a smooth real Nash algebraic function near $p$. Namely, assume that for a certain irreducible non-trivial polynomial $P(\eta, \bar{z} ; X)$, one has $P(\eta, \bar{z} ; r) \equiv \sum_{j} a_{j}(\eta, \bar{z})(r(\eta, \bar{z}))^{j} \equiv 0$ with $a_{0}(\eta, 0) \not \equiv 0$. Write $a_{0} \equiv \sum_{\alpha ;\|a\| \leq N} b_{\alpha}(\bar{z}) \eta^{\alpha}$. Then for any $z \approx p$, the compactification $\hat{Q}_{z}$ of $Q_{z}$ is understood as the variety

$$
\left\{\left[\eta_{1}, \cdots, \eta_{n+2}\right] \in \mathbb{P}^{n+1}: \sum_{\alpha=\left(\alpha_{1}, \cdots, \alpha_{n+1}\right) ;\|\alpha\|=0}^{N} b_{\alpha}(\bar{z}) \eta_{1}^{\alpha_{1}} \cdots \eta_{n+1}^{\alpha_{n+1}} \eta_{n+2}^{N-\|\alpha\|}=0 .\right\}
$$

Then the same argument shows that Lemma 3.1 holds also for domains which are locally defined by smooth Nash algebraic functions.

\section{Proof of Theorem 1.1}

We now proceed to the proof of Theorem 1.1. We first consider the case when $\gamma \subset \partial D$. Suppose that $f$ extends along $\gamma(t)$ for $0 \leq t<c \leq 1$ holomorphically, but $f$ cannot extend across $p:=\gamma(c)$. We want to find a contradiction. Notice that $f_{\gamma}(\gamma((0, c))) \subset \partial D^{\prime}$ and $\partial D^{\prime}$ is compact. By Lemma 3.1, we can easily conclude that $f_{\gamma}(\gamma(t))$ has a limit, denoted by $p^{\prime}$, as $t \rightarrow c^{-}$. Also, we can choose $U_{p}$ such that for any path $\theta$ with $\theta((0,1]) \subset U_{p} \backslash \mathcal{E} \cup \mathcal{R}(\mathcal{E})$ and $\theta(0) \in \gamma((0, c))$, the holomorphic extension of $f$ along $\theta$ takes value inside $\mathcal{P}_{p^{\prime}}^{\prime}$.

Identify $\mathbb{C}^{n+1}$ as an open subset of $\mathbb{P}^{n+1}$ in the standard way. Define $\mathcal{V}_{p} \subset \subset$ $\mathbb{C}^{n+1} \times \mathbb{C}^{n+1}$ to be the irreducible piece of $\mathcal{V}$ near $\left(p, p^{\prime}\right)$ containing the graph of 
$f$ near $(\gamma(t), f(\gamma(t)))$ with $t(<c) \approx c$. Write $\mathcal{E}_{p}$ for the genuine branching locus of the multiple-valued extension of $f$ near $p$. Namely, write $\left(\mathcal{Y}_{p}, \sigma_{p}, \mathcal{V}_{p}\right)$ for the normalization [Wh72] of $\mathcal{V}_{p}$ and write $\tilde{\pi}$ for the projection of $\mathcal{V}_{p}$ to its first copy of $\mathbb{C}^{n+1}$. Then

$$
\mathcal{E}_{p}=\tilde{\pi}\left(\sigma_{p}\left\{x \in \mathcal{Y}_{p}: x \text { is singular, or }\left.d\right|_{x} \sigma_{p} \text { is singular }\right\}\right) .
$$

We mention that $\mathcal{E}_{p}$ is of complex codimension one when it is not empty [Wh72]. We notice that $p$ must belong to $\mathcal{E}_{p}$. The proof of Theorem 1.1 will be carried out in the following three steps.

Step 1. First, by the pseudoconvexity of $D, \mathcal{E}_{p} \not \subset \bar{D}$. We will verify in this step that $\mathcal{E}_{p} \cap \mathcal{P}_{p} \cap D \cap O(p) \neq \emptyset$. Suppose not. Then the map $f$ extends holomorphically to $U_{p} \cap D$.

For any point $z \in \mathcal{P}_{p}-\left(\bar{D} \cup \mathcal{E}_{p} \cup \mathcal{S} \cup \mathcal{R}\left(\mathcal{E}_{p} \cup \mathcal{S}\right)\right)$, we can find a Jordan path $\theta(t) \in \mathcal{P}_{p}-\left(\bar{D} \cup \mathcal{E}_{p} \cup \mathcal{R}\left(\mathcal{E}_{p}\right)\right)$ for $0<t \leq 1$ with $\theta(0) \in \mathcal{P}_{p} \cap \gamma((0, c))$ and $\theta(1)=z$. Thus we get a path $\theta^{*}(t):=\mathcal{R}(\theta)(t) \in U_{p} \cap D-\mathcal{E}_{p}$ for $0<t \leq$ 1 , with $\theta^{*}(0)=\theta(0)=\gamma\left(t^{*}\right)$ and $t^{*} \in(0, c)$. The map $f$ with initial value $f_{\gamma\left(t^{*}\right)}$ can extend along both $\theta$ and $\theta^{*}$ holomorphically. Thus by (2.5), we get $f_{\theta^{*}(1)}\left(Q_{\theta(1)}^{c} \cap O\left(\theta^{*}(1)\right)\right) \subseteq Q_{f(\theta(1))}^{\prime}$, i.e., $\left.f_{\theta^{*}(1)}\left(Q_{z}^{c} \cap O\left(z^{*}\right)\right)\right) \subseteq Q_{w}^{\prime}$ for some $w \in \mathcal{P}_{p^{\prime}}^{\prime}$. Define

$$
\begin{aligned}
\mathcal{V}^{*}:=\left\{(z, w) \mid z \in \mathcal{P}_{p}-\bar{D} \cup \mathcal{E}_{p} \cup \mathcal{S} \cup \mathcal{R}\left(\mathcal{E}_{p} \cup \mathcal{S}\right),\right. \\
\left.w \in \mathcal{P}_{p^{\prime}}^{\prime} \text { and } f_{\theta^{*}(1)}\left(Q_{z}^{c} \cap O\left(z^{*}\right)\right) \subseteq Q_{w}^{\prime}\right\},
\end{aligned}
$$

which can be easily verified to be a complex subvariety of complex dimension $n+1$. Here $\theta(t)$ runs through the space of all Jordan paths as described above. Notice that $f_{\theta^{*}(1)}$ is biholomoprhic near $z^{*}=\mathcal{R}(z)$ by our arrangement of $z$.

Since $f$ extends holomorphically along paths in $U_{p} \cap \partial D-\mathcal{E}_{p}$, this $\mathcal{V}^{*}$ contains the graph of $f$ over $\left(\mathcal{P}_{p} \backslash D\right) \cap O(z)$ for any $z \in U_{p} \cap \partial D-\mathcal{E}_{p}$.

Write $\pi^{*}$ for the projection of $\mathcal{V}^{*}$ to its $z$-component. We claim that $\pi^{*-1}(z)=$ $\{(z, w)\}$ is a single point for any $z \in \mathcal{P}_{p}-\bar{D} \cup \mathcal{E}_{p} \cup \mathcal{S} \cup \mathcal{R}\left(\mathcal{E}_{p} \cup \mathcal{S}\right)$. In fact, suppose $\pi^{*-1}(z)$ contains two points $\eta_{1}=\left(z, w_{1}\right)$ and $\eta_{2}=\left(z, w_{2}\right)$ for some $z \in \mathcal{P}_{p}-\bar{D} \cup \mathcal{E}_{p} \cup \mathcal{S} \cup \mathcal{R}\left(\mathcal{E}_{p} \cup \mathcal{S}\right)$. Since $f$ is holomorphic on $U_{p} \cap D$, by the above and (2.6), $f_{\theta^{*}(1)}\left(Q_{z}^{c}\right) \subseteq Q_{w_{1}}^{\prime}$ and $f_{\sigma^{*}(1)}\left(Q_{z}^{c}\right) \subseteq Q_{w_{2}}^{\prime}$ for some paths $\theta^{*}$ and $\sigma^{*}$. Since both $w_{1}$ and $w_{2}$ are near $p^{\prime}$ and since $\sigma^{*}, \theta^{*} \subset \bar{D}$, the single value property of $f$ in $D$ near $p$ implies $Q_{w_{1}}^{\prime}$ and $Q_{w_{2}}^{\prime}$ have an open piece in common. Hence, $w_{1}=w_{2}$ by Lemma 2.2. This proves our claim.

We notice that from the way $\mathcal{V}^{*}$ is defined,

$$
\pi\left(\mathcal{V}^{*}\right) \supseteq \mathcal{P}_{p}-\bar{D} \cup \mathcal{E}_{p} \cup \mathcal{S} \cup \mathcal{R}\left(\mathcal{E}_{p} \cup \mathcal{S}\right)
$$

Hence, we can define $f(z):=w$ for any $z \in \mathcal{P}_{p}-\bar{D} \cup \mathcal{E}_{p} \cup \mathcal{S} \cup \mathcal{R}\left(\mathcal{E}_{p} \cup \mathcal{S}\right)$, where $\{(z, w)\}=\pi^{-1}(z)$. This implies that $f$ is a well defined holomorphic map in 
$\mathcal{P}_{p}-\bar{D} \cup \mathcal{E}_{p} \cup \mathcal{S} \cup \mathcal{R}\left(\mathcal{E}_{p} \cup \mathcal{S}\right)$. Notice that $f$ is bounded in $\mathcal{P}_{p}-\bar{D} \cup \mathcal{E}_{p} \cup \mathcal{S} \cup$ $\mathcal{R}\left(\mathcal{E}_{p} \cup \mathcal{S}\right)$. Since $\mathcal{E}_{p} \cup \mathcal{S} \cup \mathcal{R}\left(\mathcal{E}_{p} \cup \mathcal{S}\right)$ has Hausdorff codimension one and since $D$ is pseudoconvex of finite type, We then apply the removable singularity theorem and the Lewy-type extension theorem (say, the Trepreau theorem) to infer that $f$ extends holomorphically to $\mathcal{P}_{p}$.

Hence, $\mathcal{E}_{p}$ has to be empty near $p$. This is a contradiction.

Step 2. We will prove in this step that $\mathcal{E}_{p}$ can not cross $U_{p} \cap \partial D$ at $p$. Suppose not. Since $\mathcal{E}_{p} \cap \partial D$ then must have Hausdorff codimension 1 in $\mathcal{E}_{p}$ and most points there are smooth by Lemma 3.2, we can assume without loss of generality that $\mathcal{E}_{p}$ is smooth near $p$ and cuts $\partial D$ transversally at $p$. Since $Q_{p}$ is tangent to $\partial D$ at $p$, we hence see that by shrinking $\mathcal{P}_{p}$ if necessary $Q_{z}$ cuts $\mathcal{E}_{p}$ transversally for any $z \in \mathcal{P}_{p}$, too. Also, by nicely shrinking $U_{p}$ and $\mathcal{P}_{p}$, we may assume that the fundamental groups of $U_{p} \backslash \mathcal{E}_{p}$ and $\mathcal{P}_{p} \backslash \mathcal{E}_{p}$ are isomorphic to $\mathbb{Z}$.

By applying the removable singularities theorem and Lemma 3.1, it suffices to show that $f$ is single-valued over $\mathcal{P}_{p}-\mathcal{E}_{p} \cup \gamma([0,1]) \cup \mathcal{S} \cup \mathcal{R}\left(\mathcal{E}_{p} \cup \gamma([0,1]) \cup \mathcal{S}\right)$.

Given any $z \in \mathcal{P}_{p}-\mathcal{E}_{p} \cup \gamma([0,1]) \cup \mathcal{S} \cup \mathcal{R}\left(\mathcal{E}_{p} \cup \gamma([0,1]) \cup \mathcal{S}\right)$, let us take a smooth Jordan path $\phi$ with $\phi(t) \in \mathcal{P}_{p}-\mathcal{E}_{p} \cup \mathcal{R}\left(\mathcal{E}_{p}\right), 0<t \leq 1, \phi(0) \in$ $\partial D \cap \gamma((0, c))$ and $\phi(1)=z$. Take another Jordan loop $\sigma$ with $\sigma(t) \in \mathcal{P}_{p}-$ $\left(\mathcal{E}_{p} \cup \phi([0,1]) \cup \mathcal{R}\left(\mathcal{E}_{p} \cup \phi([0,1])\right)\right)$ for $0<t<1$ and $\sigma(0)=\sigma(1)=z$. We denote, as before, by $f_{\phi}$ the holomorphic extension of $f$ along $\phi(t)$, and $f_{\sigma}$ the holomorphic extension of $f$ along $\sigma(t)$ with initial value $f_{\phi(1)}$.

Apparently, it suffices to show that for any Jordan loop $\sigma$ as above,

$$
f_{\sigma(0)}(\sigma(0))=f_{\sigma(1)}(\sigma(1)) .
$$

Write the path $\phi^{*}(t):=\mathcal{R}(\phi)(t) \in U_{p}-\mathcal{E}_{p}(0<t \leq 1)$ with $\phi^{*}(0)=\phi(0)$ and $\phi^{*}(1)=z^{*} \in Q_{z}$. Consider the loop $\sigma^{*}(t):=\mathcal{R}(\sigma)(t) \in U_{p}-\mathcal{E}_{p}$ with $\sigma^{*}(0)=\sigma^{*}(1)=z^{*} \in Q_{z}, 0 \leq t \leq 1$. We also denote by $f_{\phi^{*}}$ the holomorphic extension of $f$ along $\phi^{*}(t)$, and by $f_{\sigma^{*}}$ the holomorphic extension of $f$ along $\sigma^{*}(t)$ with initial value $f_{\phi^{*}(1)}$. Applying Lemma 2.1 to the Jordan path $\phi \cup \sigma([0, t])$, we first see that

$$
f_{\sigma^{*}(t)}\left(O_{\sigma^{*}(t)} \cap Q_{\sigma(t)}\right) \subseteq Q_{f(\sigma(t))}^{\prime} .
$$

for $t<1$. Passing to the limit, we see (4.4) holds also for $t=1$. Recall $z=\sigma(0)=\sigma(1)$.

If we can show that $f_{\sigma^{*}(0)}\left(O_{z *} \cap Q_{z}\right)$ and $f_{\sigma^{*}(1)}\left(O_{z *} \cap Q_{z}\right)$

$$
\text { determine the germ of the same subvariety, }
$$

then from (4.4), it follows that $Q_{f(\sigma(0))}^{\prime}=Q_{f(\sigma(1))}^{\prime}$. By Lemma 2.2, we get (4.3). This would imply that $f$ is single valued in $\mathcal{P}_{p} \backslash \mathcal{E}_{p} \cup \mathcal{S} \cup \gamma([0,1]) \cup \mathcal{R}\left(\mathcal{E}_{p} \cup \gamma([0,1]) \cup\right.$ $\mathcal{S})$. Hence, to get a contradiction, it suffices to prove (4.5).

By Lemma 3.2, $Q_{z}$ intersects $\mathcal{E}_{p}$ transversely at some point $u \in Q_{z} \cap \mathcal{E}_{p} \cap U_{p}$. Thus there is a loop $\mu$ in $Q_{z}-\mathcal{E}_{p}$ such that $\mu$ represents the generator of the 
homotopic group of $U_{p}-\mathcal{E}_{p}$ with the base point $z^{*}$. Moreover, there is an integer $m$ such that $m \mu$ is homotopically equivalent to $\sigma^{*}$. For simplicity, we still use $\mu$ to denote $m \mu$. Denote, as before, by $f_{\mu(t)}$ the holomorphic extension of $f$ along $\mu(t), 0 \leq t \leq 1$, such that $f_{\mu(0)}=f_{\phi^{*}(1)}$.

Notice that $\mu(t) \in Q_{z}$ holds for all $t$ and the subvariety $Q_{z}$ is independent of $t$. By the following persistent property of the holomorphic continuation, we easily see that the subvariety $\left.f_{\mu(t)}\left(O(\mu(t)) \cap Q_{z}\right)\right)$ is always contained in $Q_{f(\mu(0))}^{\prime}$.

Fact: Let $S_{1}$ and $S_{2}$ be two complex hypersurfaces in $\mathbb{C}^{n}$ and let $F$ be a multiplevalued holomorphic map defined near $S_{1}$. Suppose that the genuine branching locus of $F$ does not contain $S_{1}$. Suppose that for a point $p \in S_{1}$, a certain branch $F^{*}$ of $F$ maps $O(p) \cap S_{1}$ into $S_{2}$. Let $\gamma$ be a path inside $S_{1}$ with $\gamma(0)=p$ (not cutting the branching locus of $F)$. Let $F^{* *}$ be the branch of $F$ near $\gamma(1)$ by holomorphically continuing $F^{*}$ along $\gamma$ to $\gamma(1)$. Then $F^{* *}\left(O_{\gamma(1)} \cap S_{1}\right) \subseteq S_{2}$.

The above fact gives that $f_{\mu(0)}\left(O_{z^{*}} \cap Q_{z}\right)$ and $f_{\mu(1)}\left(O_{z^{*}} \cap Q_{z}\right)$ determine the same subvariety. Since $f_{\mu(0)}=f_{\sigma^{*}(0)}$ and $f_{\mu(1)}=f_{\sigma^{*}(1)}$ near the point $z^{*}$, we get (4.5) and thus the property that $f(\mu(0))=f(\mu(1))$. That is, $f$ is single-valued near $p$ (away from a thin set). This contradicts the existence of the non-triviality of $\mathcal{E}_{p}$ near $p$. Therefore, Step 1 and Step 2 show that $f$ does extend across $\gamma$, whenever $\gamma \subset \partial D$.

Step 3. By what we did above, we see that for each $\gamma \subset \partial D$ with $\gamma(0)=a, f$ extends holomorphically along $\gamma$. In this step, we will show that this is the case even if $\gamma \subset \bar{D}$. Write $f_{j}=\left(f_{j}^{(1)}, \ldots, f_{j}^{(n+1)}\right)$ with $\left\{f_{j}\right\}_{j=1}^{N}$ all possible extensions by applying paths inside $\partial D$, where $N$ has to be finite by the algebraicity of $f$. Consider the following symmetric functions

$$
g^{k}(z, X):=\left(X-f_{1}^{(k)}(z)\right) \cdots\left(X-f_{N}^{(k)}(z)\right), \quad 1 \leq k \leq n+1,
$$

for any $z \in \partial D$. Write

$$
g^{k}(z, X)=X^{N}+a_{1}^{k}(z) X^{N-1}+\ldots+a_{N-1}^{k}(z) X+a_{N}^{k}(z),
$$

Obviously, by the Hartogs theorem, each coefficient is holomorphic over $\bar{D}$. Now, we can construct the graph $\mathcal{V}_{k}$ of $f_{k}$ over $D$ by using the above equation:

$$
\mathcal{V}_{k}=\left\{(z, X) \in D \times \mathbb{C}: X^{N}+a_{1}^{k}(z) X^{N-1}+\ldots+a_{N-1}^{k}(z) X+a_{N}^{k}(z)=0\right\}
$$

Notice that the number of points of each fiber, counting multiplicity, of $\mathcal{V}_{k}$ is also $N$. Suppose (1.1) is not true. For a certain $k$, the genuine branching locus of $\mathcal{V}_{k}$ is not empty and thus must be of codimension 1 . Therefore, it hits the boundary $\partial D$. This is obviously a contradiction to what we obtained in Step 1 and Step 2. The proof of Theorem 1.1 is complete. 


\section{Deformation of a path}

Let us briefly recall the Morse theory [Hir76]. Let $M$ be a smooth (real) manifold of dimension $m$ with smooth boundary, which is defined by the smooth function $\rho$. Namely, $\mathrm{d}_{\partial M} \rho \neq 0, \rho(\partial M)=0$ and $\rho(M \backslash \partial M)<0$. Let $M^{a}:=$ $\rho^{-1}(a)=\{x \in M: \rho(x)=a\}$, where $a \leq 0$. A critical point $p \in M$ of $\rho$ is called non-degenerate if the Hessian $\rho_{* *}$ is non-degenerate, i.e., $\operatorname{det}\left(\frac{\partial^{2} \rho}{\partial x^{i} \partial x^{j}}(p)\right) \neq 0$, where $\left(x^{i}\right)$ is a local coordinate system. The index of $\rho_{* *}$, over $T M_{p}$, is defined to be the maximum dimension of the subspaces of $T M_{p}$ on which $\rho_{* *}$ is positive.

Theorem 5.1. ([Hir76, pp 160, Theorem 3.3]) Let $\left\{p_{j}\right\}_{j=1}^{k}$ be a finite set of critical points of $\rho$ with indices $\left\{\lambda_{j}\right\}_{j=1}^{k}$. Assume that $\rho\left(p_{j}\right)=c$. Suppose that for a certain small $\epsilon, X_{c, \epsilon}:=\rho^{-1}([c-\epsilon, c+\epsilon])$ is compact and contains no critical points of $\rho$ other than $\left\{p_{j}\right\}$. Then there are disjoint $k \lambda_{j}$-cells $\mathcal{D}_{j} \subset$ $X_{c, \epsilon} \backslash M^{c-\epsilon}$ such that $\partial \mathcal{D}_{j} \subset M^{c+\epsilon}$, and there is a deformation retraction of $X_{c, \epsilon}$ onto $M^{c+\epsilon} \cup_{j} \mathcal{D}_{j}$.

Here, by a $k$-cell in $M$, we mean the image of a certain embedding of the closed unit $k$-ball of $\mathbb{R}^{k}$ into $M$.

To prove Corollary 1.3, we need the following lemma, which may be interesting in its own right.

Lemma 5.2. Let $D \subset \mathbb{C}^{n+1}$ be a bounded pseudoconvex domain with smooth boundary. Let $\gamma(t) \in \bar{D}$ be a path, $0 \leq t \leq 1$, such that both end points $\gamma(0), \gamma(1) \in \partial D$. Then $\gamma(t)$ can be deformed, by fixing the initial and terminal points and keeping inside $\bar{D}$, into a path $\gamma^{*} \subset \partial D$.

Proof of Lemma 5.2. Clearly, without loss of generality, we may assume that $D$ is bounded strongly pseudoconvex. It is well known that there exists a smooth strongly plurisubharmonic defining function $\rho$ of $\bar{D}$ such that all critical points of $\rho$ are non-degenerate in $D$. (This can be easily proved using the smooth approximation of bounded functions by Morse functions on compact sets of $D$ ). Clearly, $\rho$ has no critical points near $\partial D$.

Since $\rho$ is strictly plurisubharmonic, all of its non-degenerate critical points have index at least $n+1 \geq 2$.

Now we write all the critical points of $\rho$ inside $D$ as: $P_{0}, P_{1}, \ldots, P_{T}$, where each $P_{j}$ is a finite set in $\rho^{-1}\left(a_{j}\right)$ for some $a_{j}$ with $a_{0}<a_{1}<a_{2}<\ldots<a_{T}<0$. Write $\delta=\min _{j}\left\{\left|a_{j+1}-a_{j}\right| / 4,\left|a_{T}\right| / 4\right\}$ and write $\xi(\gamma)=\min _{t} \rho(\gamma(t))$. Without loss of generality, we can assume that $\xi(\gamma)>a_{1}-\delta$ and $T \geq 1$.

Let $l>0$ be such that $\xi(\gamma) \in\left[a_{l}-\delta, a_{l}+\delta\right]$. We then claim that $\gamma$ can be homopotically deformed (relative to its end points) into a path $\gamma^{*}$ with $\xi\left(\gamma^{*}\right)=$ $a_{l+1}+\delta$. Indeed, by Theorem 5.1 , there is a deformation retraction from $\rho^{-1}\left(\left[a_{l}-\right.\right.$ $\delta, 0])$ to $\rho^{-1}\left(\left[a_{l}+\delta, 0\right]\right) \cup_{j} \mathcal{D}_{j}$, where the mutually disjointed $\mathcal{D}_{j}^{\prime} s$ are certain embedded $\lambda_{j}$-cells with $\lambda_{j} \geq 2, \mathcal{D}_{j} \subset \rho^{-1}\left(\left[a_{l}+\delta, a_{l}-\delta\right)\right)$ and $\partial \mathcal{D}_{j} \subset M^{a_{l}+\delta}$. Apparently, we can assume that $\gamma$ is smooth and thus $\mathcal{D}_{j} \backslash\left(\partial \mathcal{D}_{j} \cup \gamma^{*}\right) \neq \emptyset$. For each $j$, take $q_{j} \in \mathcal{D}_{j} \backslash\left(\partial \mathcal{D}_{j} \cup \gamma^{*}\right)$. Notice that $\rho^{-1}\left(\left[a_{l}+\delta, 0\right]\right) \cup_{j}\left(\mathcal{D}_{j} \backslash\left\{p_{j}\right\}\right)$ can be 
homotopically retracted to $\rho^{-1}\left(\left[a_{l}+\delta, 0\right]\right) \cup_{j} \partial \mathcal{D}_{j}=\rho^{-1}\left(\left[a_{l}+\delta, 0\right]\right)$. Therefore, combining these deformations with $\gamma$, we see that $\gamma$ can be deformed into a path $\gamma^{*}$ with $\xi\left(\gamma^{*}\right)=a_{l}+\delta$. Applying the easy case of Theorem 5.1 (the case with empty critical points), we can further assume that $\xi\left(\gamma^{*}\right)=a_{l+1}-\delta$ if $T>1$. This completes the proof of the claim.

Finally, an inductive use of the above argument shows that $\gamma$ can be deformed into a tubular neighborhood $\rho^{-1}\left(\left[0, a_{T}+\delta\right]\right)$ of $\partial D$ and thus $\partial D$.

\section{Proof of Theorem 1.2}

Proof of Corollary 1.3. The local biholomorphic property follows by applying Theorem 1.1 to $f$ and $f^{-1}$. It remains to prove (1.2). Suppose (1.2) is not true. Then there is some $c, 0<c \leq 1$, such that $f(\gamma(t)) \in D^{\prime}$ for $0<t<c$ but $f(\gamma(c)) \in \partial D^{\prime}$.

Denote by $\gamma^{\prime}(t):=f(\gamma(c t)), 0 \leq t \leq 1$, the pushforward path. Then $\gamma^{\prime}(0), \gamma^{\prime}(1) \in \partial D^{\prime}$ and $\gamma^{\prime}(t) \in D^{\prime}$ for $0<t<1$. By Lemma 5.2, the path $\gamma^{\prime}$ can be deformed, via a continuous family of paths $\left\{\gamma_{s}^{\prime}\right\}_{s}$ into a path $\gamma^{*} \subset \partial D^{\prime}$ with $\gamma^{*}(0)=\gamma^{\prime}(0)$ and $\gamma^{*}(1)=\gamma^{\prime}(1)$. Here $\gamma_{s}^{\prime}(0)=\gamma^{\prime}(0), \gamma_{s}^{\prime}(1)=\gamma^{\prime}(1)$ and $\gamma_{s}^{\prime}(t) \in \bar{D}^{\prime}$. Consider the local inverse $f^{-1}$ near $a^{\prime}:=f(a)$. Since $\partial D$ is strongly pseudoconvex, we can apply Theorem 1.1 to extend $f^{-1}$ along each $\gamma_{s}^{\prime}$. Since the extension of $f^{-1}$ along $\gamma^{*}$ takes the value in $\partial D$, by the monodromy theorem, we get that $\gamma(c) \in \partial D$, contradicting the above assumption.

Proof of Corollary 1.4. (i) When $D$ is simply connected, by Corollary 1.3, $f$ extends to a locally biholomorphic map $f: D \rightarrow D^{\prime}$. Since $f(\partial D) \subset \partial D^{\prime}, f$ must be a proper map. Hence, $f$ is a covering map. (ii) follows from (i).

Proof of Theorem 1.2. Since $\partial D$ is spherical, there exists a local biholomorphic map $f$ from $\partial \mathbb{B}^{n+1}$ to $\partial D$. From Corollary 1.4(i), $f$ extends to a universal covering map $f: \mathbb{B}^{n+1} \rightarrow D$. Hence, $D$ is biholomorphic to $\mathbb{B}^{n+1} / \Gamma$, where $\Gamma \subset \operatorname{Aut}\left(\mathbb{B}^{n+1}\right)$ is isomorphic to the deck transformation group of the above mentioned covering $f$. By the properness of $f$, we see that $\Gamma$ is finite. However, by a classical theorem of E. Cartan, it is known that any finite subgroup of Aut $\left(\mathbb{B}^{n+1}\right)$ must have a common fixed point. (See [Ru80] [BS77], for example). This contradicts the smoothness of $D$.

Remark 6.1. (a) Taking into consideration of Remark 3.3 (b), by exactly the same argument in the paper, it is clear that Theorem 1.1 and Theorem 1.2 also hold when $D$ and $D^{\prime}$ are merely assumed to be bounded smooth Nash algebraic domains, those which can be locally defined by (smooth) Nash algebraic functions, in the sense of Remark 3.3 (b).

(b) By investigating the proof, one sees easily that Theorem 1.1 also holds when $D^{\prime}$ is just assumed to be weakly pseudoconvex but with the following geometric property: For any $p^{\prime} \in \partial D^{\prime}$, the $A_{z^{\prime}}^{\prime}$ as defined in (2.2) is singlevalued whenever $z^{\prime} \approx p^{\prime}$. Hence, Theorem 1.2 also holds in the following more general setting: 
Theorem 1.2'. Let $D$ be a bounded Nash algebraic domain (in the sense of Remark 3.3 (b)) such that (i) $\partial D$ is connected and has at least one spherical point; (ii) for any $p \in \partial D$, the $A_{z}$ defined as in (2.2) is single valued for $z \approx p$. Then $D$ is biholomorphic to the ball.

Indeed, let $D$ be as above and let $a \in \partial D$ be a spherical point. Then there is a local biholomorphic map $f$ defined near $a$ such that $f$ maps $\partial D$ near $a$ into $\partial \mathbb{B}^{n+1}$. By the argument in the proof of Lemma 3.2, it follows that $D$ must be pseudoconnvex. Consider the extension of $f^{-1}$. We then also similarly show that $D$ is covered by the ball and thus must be biholomorphic to the ball.

\section{Acknowledgements}

The authors wish to thank S. Baouendi, S. S. Chern, P. Ebenfelt, B. Shiffman, L. Rothschild and S. Webster for their interest to this work. They are indebted to S. Webster for his many useful conversations related to this work, in particular, for his help with Lemma 5.2.

\section{References}

[A74] H. Alexander Holomorphic mappings from the ball and polydisc, Math. Ann. 209 (1974), 249-256.

[BER96] M. S. Baouendi, P. Ebenfelt and L. P. Rothschild, Algebraicity of holomorphic mappings between real algebraic sets in $\mathbb{C}^{n}$, Acta Math. 177 (1996), 225-273.

[BHR96] M. S. Baouendi, X. Huang and L. P. Rothschild, Regularity of CR mappings between algebraic hypersurfaces, Invent Math. 125 (1996), 13-36.

[BR95] M. S. Baouendi and L. P. Rothschild, Mappings of real algebraic hypersurfaces,, J. Amer. Math. Soc. 8 (1995), 997-1015.

[Be90] S. Bell, Mapping problems in complex analysis and the $\bar{\partial}$-problem,, Bull. Amer. Math. Soc. 22 (1990), 233-259.

[BS76] D. Jr Burns and S. Shnider, Spherical hypersurfaces in complex manifolds, Invent. Math. 33 (1976), 223-246.

[CJ96] S.-S. Chern and S. Ji, On the Riemann mapping theorem,, Ann. of Math. 144 (1996), 421-439.

[DF77] K. Diederich and J. Fornaess, Pseudoconvex domains: bounded strictly plurisubharmonic exhaustion functions, Invent. Math. 39 (1977), 129-141.

[DFY94] K. Diederich J. Fornaess, and Z. Ye, Biholomorphisms in dimension 2,, J. Geome. Anal. 49 (1994), 539-552.

[E96] P. Ebenfelt, On the unique continuation problem for $C R$ mappings into nonminimal hypersurfaces, J. Geom. Anal. 6 (1996), 385-405.

[Hu94] X. Huang, On the mapping problem for algebraic real hypersurfaces in the complex spaces of different dimensions, Ann. Inst. Fourier, Grenoble 44 (1994), 433-463.

[Hu94T] _ Geometric analysis in several complex variables, Chapter 2, Ph. D. Thesis, Washington University, August, 1994.

[Hu96] Schwarz reflection principle in complex spaces of dimension two, Comm. Partial Diff. Eq. 21 (1996), 1781-1828.

[Hir76] M. Hirsch, Differential topology, Grad. Texts in Math., 33, Springer-Verlag, New York-Heidelberg, 1976.

[Pi78] S. Pinchuk, On holomorphic maps of real-analytic hypersurfaces,, Mat. Sb., Nov. Ser 105 (1978), 574-593. 
[Po07] H. Poincaré, Les fonctions analytiques de deux variables et la représentation conforme, Rend. Circ. Mat. Palermo (1907), 185-220.

[Ru80] W. Rudin, Function theory in the unit ball of $\mathbb{C}^{n}$, Grundlehren Math. Wiss., 241, Springer-Verlag, New York, 1980.

[Ro80] J. P. Rosay, Sur une characterization de la boule parmi les domains de $\mathbb{C}^{n}$ par son groupe d'automorphismes., Ann. Inst. Fourier. Grenoble 26 (1980), 805-809.

[Ta62] N. Tanaka, On pseudo-conformal geometry of hypersurfaces of the space of $n$ complex variables, J. Math. Soc. Japan 14 (1962), 397-429.

[VEK86] A. G. Vitushkin, V. V. Ezhov, and N. G. Kruzhilin, Extension of holomorphic mappings along real-analytic hypersurfaces, Proc. Steklov Inst. Math. 167 (1986), 63-102.

[We77] S. M. Webster, On the mapping problem for algebraic real hypersurfaces, Invent. Math. 43 (1977), 53-68.

[We79] , Biholomorphic mappings and the Bergman kernel off the diagonal, Invent. Math. 51 (1979), 155-169.

[Wel82] R. O. Jr. Wells, The Cauchy-Riemann equations and differential geometry, Bull. Amer. Math. Soc. 6 (1982), 187-198.

[Wh72] H. Whitney, Complex analytic varieties (1972), Addison-Wesley Publishing Company, Reading, Mass.-London-Don Mills, Ont..

[Wo77] B. Wong, Characterization of the unit ball in $\mathbb{C}^{n}$ by its automorphism groups, Invent. Math. 41 (1977), 253-257.

Department of Mathematics, Rutgers University, New Brunswick, NJ 08903 USA

Department of Mathematics, University of Houston, Houston, TX 77204 USA 